\title{
Delocalization In Platinum-Alkynyl Systems: A Metal-Bridged Organic Mixed- Valence Compound
}

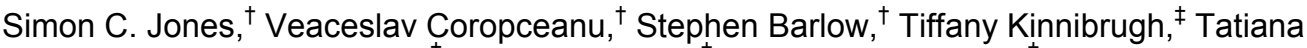
Timofeeva, ${ }^{\ddagger}$ Jean-Luc Brédas ${ }^{\dagger}$ and Seth R. Marder ${ }^{*,}$

${ }^{\dagger}$ School of Chemistry and Biochemistry, Georgia Institute of Technology, Atlanta, GA 30332, USA, and

${ }^{7}$ Department of Natural Sciences, New Mexico Highlands University, Las Vegas, NM 87701, USA

\section{Supporting Information}

\section{Experimental Details}

1: A slurry of (4,4'-dimethoxyphenyl,4"-ethynyl)triphenylamine (1.30 g, $3.95 \mathrm{mmol})$ and transdichlorobis(triethylphosphine)platinum(II) $(0.99 \mathrm{~g}, 1.97 \mathrm{mmol})$ was stirred in $\mathrm{Et}_{2} \mathrm{NH}(25 \mathrm{ml})$, affording a pale yellow precipitate immediately. This mixture was refluxed for $16 \mathrm{hr}$, volatiles were removed in vacuo and the resulting brown solid was washed with $50 \mathrm{ml}$ water. This aqueous slurry was extracted with $\mathrm{CH}_{2} \mathrm{Cl}_{2}(2 \times 50 \mathrm{ml})$ to give a dark brown solution which was dried over $\mathrm{Na}_{2} \mathrm{SO}_{4}$. Subsequent filtration and removal of volatiles from the filtrate in vacuo afforded a brown solid which was purified by column chromatography (silica gel, $\mathrm{CH}_{2} \mathrm{Cl}_{2}$ elution) to give $1(1.10 \mathrm{~g}, 1.01 \mathrm{mmol}, 51 \%)$ as a pale yellow crystalline solid. Single crystals suitable for X-ray crystallography were grown by slow-cooling a solution in $1: 1 \mathrm{v} / \mathrm{v} \mathrm{EtOH} /$ acetone to $-10{ }^{\circ} \mathrm{C}$.

Analysis found (calculated) \%: C 61.65 (61.81) H 6.07 (6.11) N 2.60 (2.57); ${ }^{1} \mathrm{H} \mathrm{NMR}\left(\mathrm{CDCl}_{3}\right.$, $250 \mathrm{MHz}, 298 \mathrm{~K}): \delta 7.07(\mathrm{~d}, J=9 \mathrm{~Hz}, 4 \mathrm{H}), 6.99(\mathrm{~d}, J=9 \mathrm{~Hz}, 8 \mathrm{H}), 6.79-6.75$ (2 overlapping d, $J=9 \mathrm{~Hz}, 12 \mathrm{H}), 3.77(\mathrm{~s}, 12 \mathrm{H}), 2.14(\mathrm{~m}, 12 \mathrm{H}), 1.18(\mathrm{~m}, 18 \mathrm{H}) ;{ }^{31} \mathrm{P}\left\{{ }^{1} \mathrm{H}\right\} \mathrm{NMR}\left(\mathrm{CDCl}_{3}, 121 \mathrm{MHz}\right.$, $298 \mathrm{~K}): \delta 11.49\left(\mathrm{~s},{ }^{1} J_{\mathrm{P}-\mathrm{Pt}}=2383 \mathrm{~Hz}\right) ;{ }^{13} \mathrm{C}\left\{{ }^{1} \mathrm{H}\right\} \mathrm{NMR}\left(\mathrm{CDCl}_{3}, 100 \mathrm{MHz}, 298 \mathrm{~K}\right): \delta 155.5(\mathrm{~s}, \underline{C O}$ quat.), 145.9 (s, $\underline{C} \mathrm{~N}$ quat.), 141.2 (s, $\underline{C} \mathrm{~N}$ quat), 131.5 (s, $\underline{C} \mathrm{H}$ aromatic), 126.0 (s, $\underline{C H}$ aromatic), 121.4 (s, $\underline{C}$ quat), 121.0 (s, $\underline{C} \mathrm{H}$ aromatic), 114.6 (s, $\underline{C H}$ aromatic), $108.7\left(\mathrm{~s},{ }^{2} \mathrm{~J}_{\mathrm{C}-\mathrm{Pt}}=265 \mathrm{~Hz}, \underline{C}\right.$ alkyne), $105.2\left(\mathrm{t},{ }^{2} \mathrm{~J}_{\mathrm{C}-\mathrm{P}}=15 \mathrm{~Hz},{ }^{1} \mathrm{~J}_{\mathrm{C}-\mathrm{Pt}}=970 \mathrm{~Hz}, \underline{C}\right.$ alkyne), 55.5 (s, $\underline{C} \mathrm{H}_{3}$ methoxy), 16.3 (vt, $\left.{ }^{1} J_{\mathrm{C}-\mathrm{P}}=17 \mathrm{~Hz}, \underline{C} \mathrm{H}_{2}\right), 8.3$ (s, $\underline{C H}_{3}$ phosphine); MS (MALDI-TOF): $1087\left(\mathrm{M}^{+}\right)$; IR (KBr): 2100 $\mathrm{cm}^{-1}\left(v_{\mathrm{CC}}\right) ; \mathrm{CV}$ (vs. ferrocenium/ferrocene, $\left.\mathrm{CH}_{2} \mathrm{Cl}_{2} / 0.1 \mathrm{M}{ }^{\mathrm{n}} \mathrm{Bu}_{4} \mathrm{NPF}_{6}, 298 \mathrm{~K}\right):+0.09 \mathrm{~V}$ (reversible), + $0.15 \mathrm{~V}$ (reversible), $+0.75 \mathrm{~V}$ (quasi-reversible), + $0.87 \mathrm{~V}$ (quasi-reversible)

Crystal data for 1: pale yellow, $\mathrm{C}_{56} \mathrm{H}_{66} \mathrm{~N}_{2} \mathrm{O}_{4} \mathrm{P}_{2} \mathrm{Pt}, M=1088.16$, triclinic, space group $P \overline{1}(2)$, $a=9.8506(19), b=11.529(2), c=11.5728(15) \AA, \alpha=85.452(4), \beta=76.899(3), \gamma=86.067(3)^{\circ}$, $V=1274.4(4) \AA^{3}, T=120(2) \mathrm{K}, Z=1, F(000)=556, \mu=2.862 \mathrm{~mm}^{-1}$. Data were collected in the 
$\theta$ range $1.77-28.11^{\circ}, 12841$ reflections, 6009 unique $\left(R_{\text {int }}=0.0561\right)$. The structure was solved by direct methods and refined by full-matrix least-squares techniques against $F^{2} ; R_{1}=0.0578$, $\mathrm{w} R_{2}=0.1129, \mathrm{GOF}=0.945$ (all data) with allowance for thermal anisotropy of all non-hydrogen atoms; max. residual electron density 1.809 e $\AA^{-3}$.

2 was synthesised by the literature method. ${ }^{1}$

\section{Computational details}

All calculations were performed with the Gaussian-98 program (Revision A.9) ${ }^{2}$ at the B3LYP level using a LANL2DZ basis set and corresponding effective core pseudopotential. The experimental crystal structure was used for TD-DFT calculations.

\section{Absorption and luminescence spectra for 1 in $\mathrm{CH}_{2} \mathrm{Cl}_{2}$}

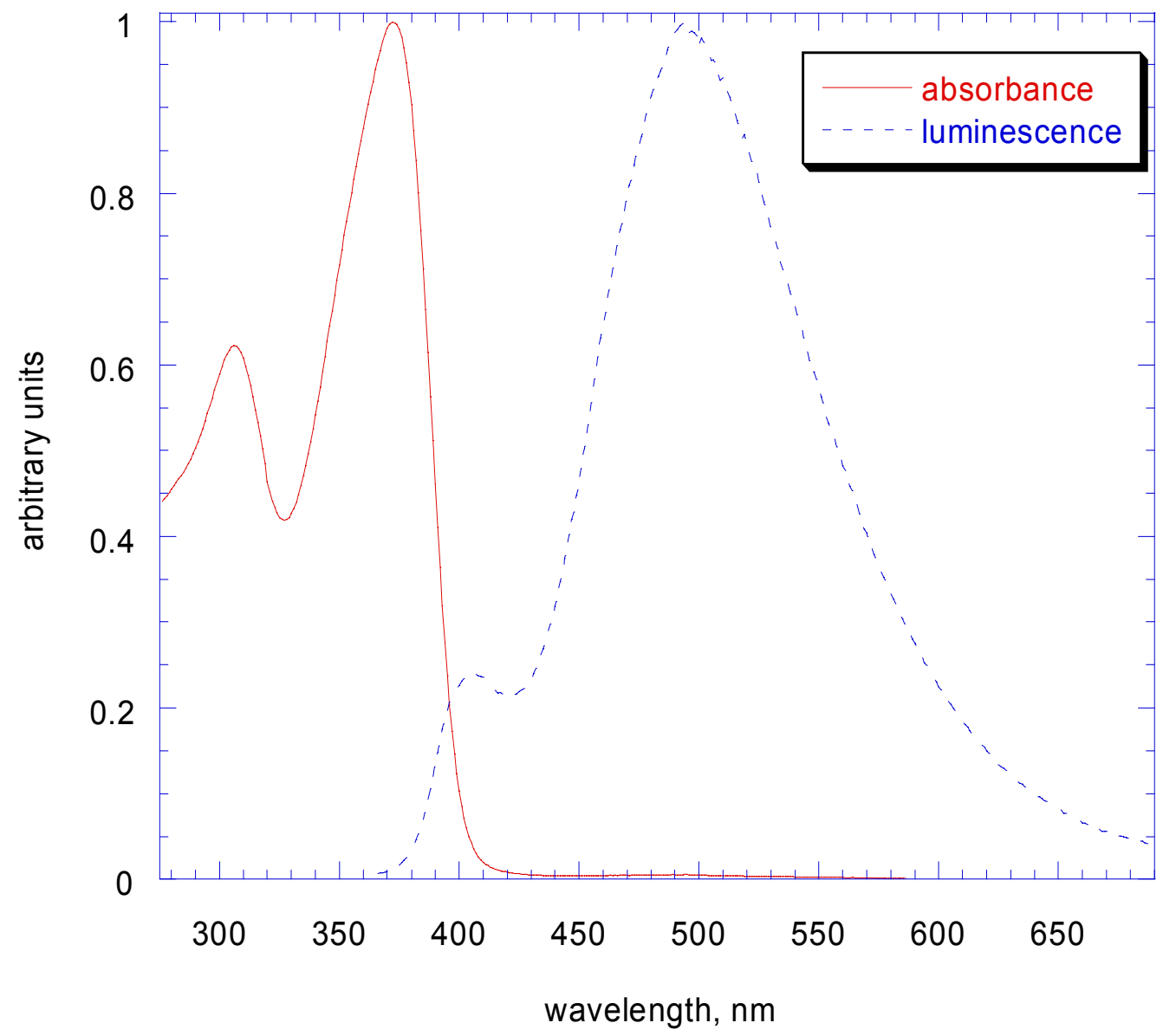




\section{Voltammetry of 1}
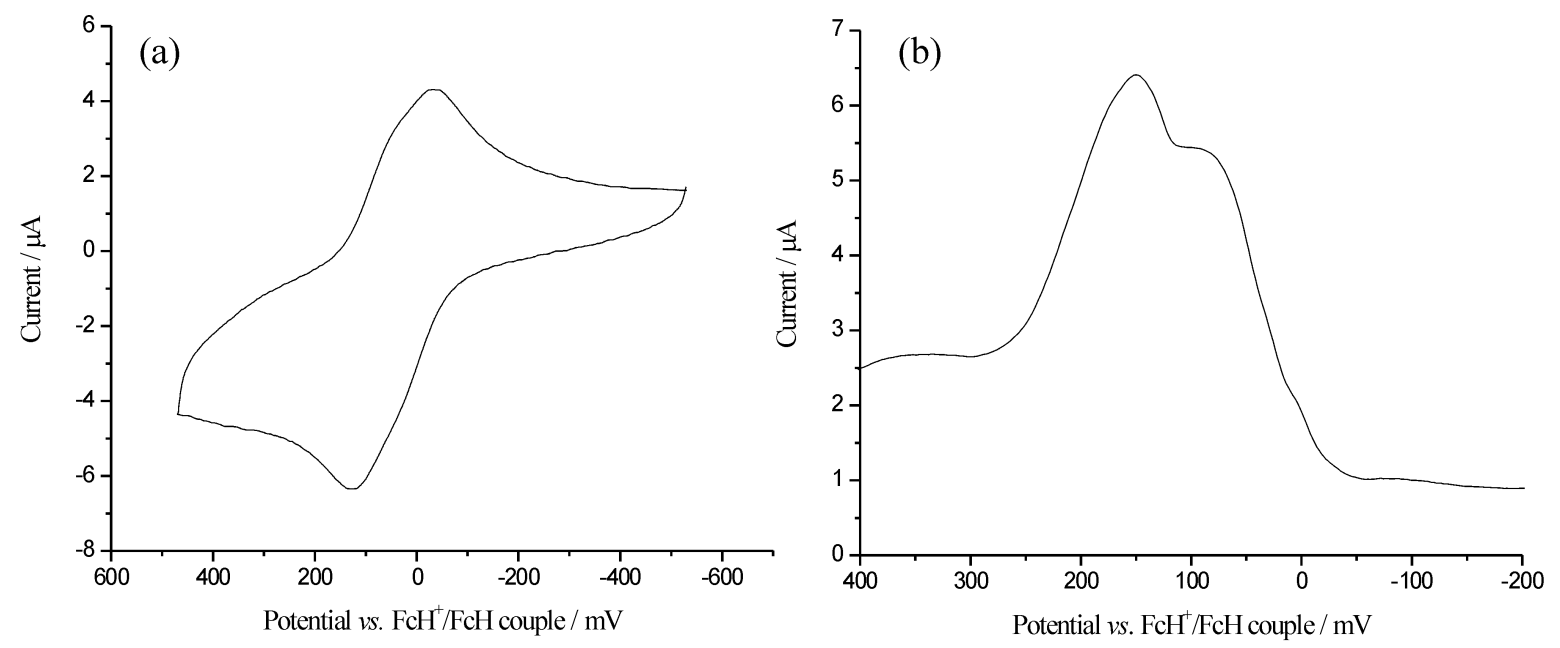

(a) Cyclic voltammogram of $1\left(\mathrm{CH}_{2} \mathrm{Cl}_{2} / 0.1 \mathrm{M}^{\mathrm{n}} \mathrm{Bu}_{4} \mathrm{NPF}_{6}, 298 \mathrm{~K}, 50 \mathrm{mV} \mathrm{s}{ }^{-1}\right)$; (b) Differential pulse voltammogram of $1\left(\mathrm{CH}_{2} \mathrm{Cl}_{2} / 0.1 \mathrm{M}^{\mathrm{n}} \mathrm{Bu}_{4} \mathrm{NPF}_{6}, 298 \mathrm{~K}\right.$, scan rate $10 \mathrm{mV} \mathrm{s}{ }^{-1}$, sample width $=17 \mathrm{~ms}$, pulse amplitude $=40 \mathrm{mV}$, pulse width $=50 \mathrm{~ms}$, pulse period $=200 \mathrm{~ms}$ ).

\section{EPR spectroscopy of 1}
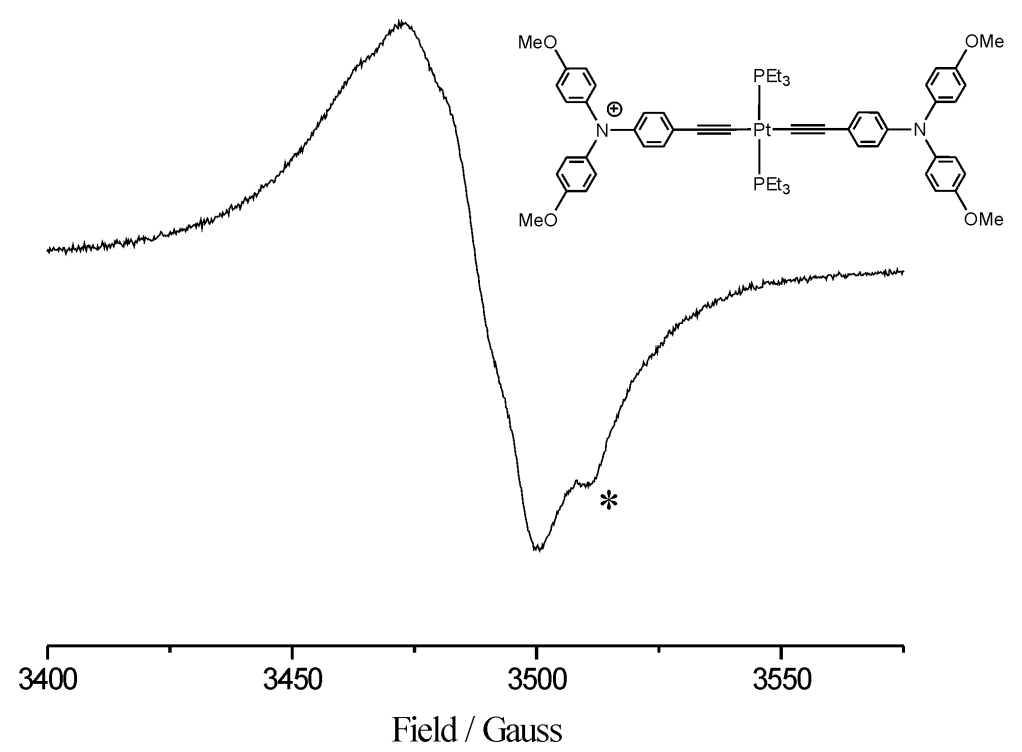

EPR of $[1]^{+}$in $\mathrm{CH}_{2} \mathrm{Cl}_{2}$ at $298 \mathrm{~K}$. A broad three-line spectrum is observed with $g=2.018$, $a_{\mathrm{N}}=9.3 \mathrm{G}$; the peak marked with an asterisk increases in intensity with time and is presumably due to a decomposition product. This spectrum indicates that the first oxidation of $\mathbf{1}$ is N-based 
(compare $g=2.003, a_{\mathrm{N}}=10.2 \mathrm{G}$ for $\mathrm{Ph}_{3} \mathrm{~N}^{+}, a_{\mathrm{N}}=9.1 \mathrm{G}$ for $\left.\left(p-\mathrm{MeOC}_{6} \mathrm{H}_{4}\right)_{3} \mathrm{~N}^{+}\right){ }^{3,4}$ The spectra of simple triarylaminum cations obtained by us under similar conditions are likewise broad.

\section{References}

1 Lambert, C.; Nöll, G. J. Am. Chem. Soc. 1999, 121, 8434-8442.

2 Frisch, M. J.; Trucks, G. W.; Schlegel, H. B.; Scuseria, G. E.; Robb, M. A.; Cheeseman, J. R.; Zakrzewski, V. G.; Montgomery, J. A., Jr.; Stratmann, R. E.; Burant, J. C.; Dapprich, S.; Millam, J. M.; Daniels, A.D.; Kudin, K. N.; Strain, M. C.; Farkas, O.; Tomasi, J.; Barone, V.; Cossi, M.; Cammi, R.; Mennucci, B.; Pomelli, C.; Adamo, C.; Clifford, S.; Ochterski, J.; Petersson, G. A.; Ayala, P. Y.; Cui, Q.; Morokuma, K.; Malick, D. K.; Rabuck, A. D.; Raghavachari, K.; Foresman, J. B.; Cioslowski, J.; Ortiz, J. V.; Baboul, A. G.; Stefanov, B. B.; Liu, G.; Liashenko, A.; Piskorz,P.; Komaromi, I.; Gomperts, R.; Martin, R. L.; Fox, D. J.; Keith, T.; Al-Laham, M. A.; Peng, C. Y.; Nanayakkara, A.; Challacombe, M.; Gill, P. M. W.; Johnson, B.; Chen, W.; Wong, M. W.; Andres, J. L.; Gonzalez, C.; Head-Gordon, M.; Replogle, E. S.; Pople, J. A. Gaussian 98, Revision A.9; Gaussian, Inc.; Pittsburgh, PA, 1998.

3 Bamberger, S.; Hellwinkel, D.; Neugebauer, F. A. Chem. Ber. 1975, 108, 2416-2421.

4 Seo, E. T.; Nelson, R. F.; Fritsch, J. M.; Marcoux, L. S.; Leedy, D. W.; Adams, R. N. J. Am. Chem. Soc. 1966, 88, 3498-3503. 